International Journal of Pure and Applied Mathematics

Volume 104 No. 2 2015, 227-236

ISSN: 1311-8080 (printed version); ISSN: 1314-3395 (on-line version)

url: http://www.ijpam.eu

doi: http://dx.doi.org/10.12732/ijpam.v104i2.7

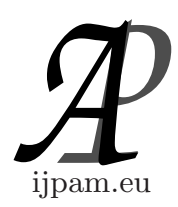

\title{
WEAKLY WO-CONTINUOUS FUNCTIONS ON ASSOCIATED $w$-SPACES
}

\author{
Young Key Kim${ }^{1}$, Won Keun $\mathrm{Min}^{2} \S$ \\ ${ }^{1}$ Department of Mathematics \\ MyongJi University \\ Youngin, 449-728, KOREA \\ ${ }^{2}$ Department of Mathematics \\ Kangwon National University \\ Chuncheon, 200-701, KOREA
}

Abstract: We introduce the notions of weakly $W O$-continuity and strongly $W$-closed graph.

We study some characterizations and properties of such functions.

AMS Subject Classification: 54A05, 54B10, 54C10, 54D30

Key Words: weakly $W O$-continuous, $W$-compact, $w$ - $T_{2}$-space, strongly $W$ closed graph

\section{Introduction}

In [17], Siwiec introduced the notions of weak neighborhoods and weak base in a topological space. We introduced the weak neighborhood systems defined by using the notion of weak neighborhoods in [12]. And we also introduced a

Received: June 10, 2015

(C) 2015 Academic Publications, Ltd.

$\S_{\text {Correspondence author }}$ 
weak neighborhood space (briefly WNS) which is independent of neighborhood spaces [4] and general topological spaces [2]. We introduced the notion of $w$ spaces in [13] and investigated some basic properties. In [14], we introduced and studied the notions $W K$-continuity and $W O$-continuity on associated $w$-spaces. In this paper, we introduce the notions of weakly $W O$-continuity and strongly $W$-closed graph on associated $w$-spaces. And we study some characterizations and properties of such functions.

\section{Preliminaries}

Let $S$ be a subset of a topological space $X$. The closure (resp., interior) of $S$ will be denoted by $\operatorname{cl} S$ (resp., int $S$ ). A subset $S$ of $X$ is called a preopen set [10] (resp., $\alpha$-set [14], semi-open [6]) if $S \subset \operatorname{int}(c l(S)$ ) (resp., $S \subset$ $\operatorname{int}(\operatorname{cl}(\operatorname{int}(S))), S \subset \operatorname{cl}(\operatorname{int}(S)))$. The complement of a preopen set (resp., $\alpha$ set, semi-open) is called a preclosed set (resp., $\alpha$-closed set, semi-closed). The family of all preopen sets (resp., $\alpha$-sets, semi-open sets) in $X$ will be denoted by $P O(X)$ (resp., $\alpha(X), S O(X)$ ). We know the family $\alpha(X)$ is a topology finer than the given topology on $X$.

A subset $A$ of a topological space $(X, \tau)$ is said to be:

(a) $g$-closed [5] if $C l(A) \subset U$ whenever $A \subset U$ and $U$ is open in $X$;

(b) $g p$-closed [7] if $p C l(A) \subset U$ whenever $A \subset U$ and $U$ is open in $X$;

(c) gs-closed $[1,3]$ if $s C l(A) \subset U$ whenever $A \subset U$ and $U$ is open in $X$;

(d) $g \alpha$-closed [9] if $\tau^{\alpha} C l(A) \subset U$ whenever $A \subset U$ and $U$ is $\alpha$-open in $X$, where $\tau^{\alpha}=\alpha(X)$;

(e) $g \alpha^{*}$-closed [8] if $\tau^{\alpha} C l(A) \subset \operatorname{Int}(U)$ whenever $A \subset U$ and $U$ is $\alpha$-open in $X$;

(f) $g \alpha^{* *}$-closed [8] if $\tau^{\alpha} C l(A) \subset \operatorname{Int}(C l(U))$ whenever $A \subset U$ and $U$ is $\alpha$-open in $X$;

(g) $\alpha g$-closed [9] if $\tau^{\alpha} C l(A) \subset U$ whenever $A \subset U$ and $U$ is open in $X$;

(h) $\alpha^{* *} g$-closed [9] if $\tau^{\alpha} C l(A) \subset \operatorname{Int}(C l(U))$ whenever $A \subset U$ and $U$ is open in $X$;

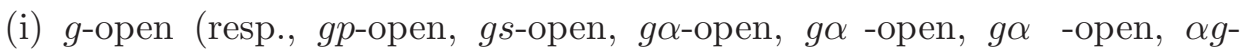
open;

$\alpha^{* *} g$-open) if the complement $A$ is $g$-closed (resp., gp-closed, $g s$-closed, $g \alpha$ closed, $g \alpha^{*}$-closed, $g \alpha^{* *}$-closed, $\alpha g$-closed, $\alpha^{* *} g$-closed). 
The family of all $g$-open (resp., gp-open, $g s$-open, $g \alpha$-open, $g \alpha^{*}$-open, $g \alpha^{* *}$-open, $\alpha g$-open, $\alpha^{* *} g$-open) sets in $X$ will be denoted by $g O(X)$ (resp., $g p O(X), g s O(X), g \alpha O(X), g \alpha^{*} O(X), g \alpha^{* *} O(X), \alpha g O(X), \alpha^{* *} g O(X)$.

Definition $2.1([13])$. Let $X$ be a nonempty set. A subfamily $w_{X}$ of the power set $P(X)$ is called a weak structure on $X$ if it satisfies the following:

(1) $\emptyset \in w_{X}$ and $X \in w_{X}$.

(2) For $U_{1}, U_{2} \in w_{X}, U_{1} \cap U_{2} \in w_{X}$.

Then the pair $\left(X, w_{X}\right)$ is called a $w$-space on $X$. Then $V \in w_{X}$ is called a $w$-open set and the complement of a $w$-open set is a $w$-closed set.

Let $(X, \tau)$ be a topological space. Then the family $\tau, G O(X), g \alpha O(X)$, $g \alpha^{*} O(X), g \alpha^{* *} O(X), \alpha g O(X)$ and $\alpha^{* *} g O(X)$ on $X$ are all weak structures on $X$. But $P O(X), G P O(X)$ and $S O(X)$ are not weak structures on $X$.

Definition $2.2([13])$. Let $\left(X, w_{X}\right)$ be a $w$-space. For a subset $A$ of $X$, the $w$-closure of $A$ and the $w$-interior of $A$ are defined as the following:

(1) $w C l(A)=\cap\left\{F: A \subset F, X-F \in w_{X}\right\}$.

(2) $w \operatorname{Int}(A)=\cup\left\{U: U \subset A, U \in w_{X}\right\}$.

Theorem $2.3([13])$. Let $\left(X, w_{X}\right)$ be a $w$-space and $A \subset X$.

(1) $x \in w \operatorname{Int}(A)$ if and only if there exists a $w$-open subset $U$ containing $x$ such that $U \subset A$.

(2) $x \in w C l(A)$ if and only if $A \cap V \neq \emptyset$ for every $w$-open subset $V$ containing $x$.

Let $X$ be a nonempty set and let $(X, \tau)$ be a topological space. A subfamily $w_{\tau}$ of the power set $P(X)$ is called an associated weak structure [14] on $X$ if $\tau \subset w_{\tau}$. Then the pair $\left(X, w_{\tau}\right)$ is called an associated $w$-space with $\tau$ [14].

Definition $2.4([14])$. Let $f:\left(X, w_{\tau}\right) \rightarrow(Y, \mu)$ be a function on an associated $w$-space $X$ with $\tau$ and a topological space $(Y, \mu)$. Then $f$ is said to be:

(1) WO-continuous if for $x \in X$ and for each open subset $V$ containing $f(x)$, there is a $w$-open subset $U$ of $X$ containing $x$ such that $f(U) \subset V$,

(2) WK-continuous if for every open set $V$ in $Y, f^{-1}(V)$ is a $w$-open set in $X$.

Remark 2.5. Every $W K$-continuous function is a $W O$-continuous function. But the converse may not be true as shown in Example 4.6 [14]. 
Definition $2.6([15])$. Let $\left(X_{i}, w_{i}\right)$ be a $w$-space for $i \in J$. Set $\mathbf{S}=$ $\left\{\pi_{i}^{-1}\left(U_{i}\right): U_{i} \in w_{i}\right.$ for $\left.i \in J\right\}$ where $\pi_{i}: \prod X_{i} \rightarrow X_{i}$ is an $i$-th projection map. We call $\mathbf{W}=\{\cap \mathbf{B}: \mathbf{B} \subseteq \mathbf{S}$ and $\mathbf{B}$ is finite $\}$ the product weak structure on $X=\prod X_{i}$, and $\left(\prod X_{i}, \mathbf{W}\right)$ is called the product weak space (briefly, product $w$-space).

\section{Weakly WO-Continuous Functions}

Definition 3.1. Let $\left(X, w_{\tau}\right)$ be an associated $w$-space and $(Y, \mu)$ be a topological space. Then $f: X \rightarrow Y$ is said to be weakly WO-continuous if for $x \in X$ and for each open subset $V$ containing $f(x)$, there is a $w$-open subset $U$ containing $x$ such that $f(U) \subset C l(V)$.

From Remark 2.5 and definition of weakly $W O$-continuity, we get the following implications but the converses are not true:

continuous $\Rightarrow W K$-continuous $\Rightarrow W O$-continuous $\Rightarrow$ weakly WO-continuous.

Example 3.2. Let $X=Y=\{a, b, c\}$. Consider an associated weak structures $w_{\sigma}=\{\emptyset,\{a\},\{c\}, X\}$ and a topological space $\mu=\{\emptyset,\{a, b\}, Y\}$.

Let $f:\left(X, w_{\sigma}\right) \rightarrow(Y, \mu)$ be a function defined by $f(x)=x$, for $x \in X$. Then since $C l(\{a, b\})=Y, f$ is weakly $W O$-continuous. But $f$ is not $W O$-continuous because $X$ is the only $w_{\sigma}$-open set containing $b$ in $X$.

Theorem 3.3. Let $f:\left(X, w_{\sigma}\right) \rightarrow(Y, \mu)$ be a function on an associated $w$-space $\left(X, w_{\sigma}\right)$ and a topological space $(Y, \mu)$. Then the following statements are equivalent:

(1) $f$ is weakly $W O$-continuous.

(2) $f^{-1}(V) \subseteq w \operatorname{Int}\left(f^{-1}(C l(V))\right)$ for every open subset $V$ of $Y$.

(3) $w C l\left(f^{-1}(\operatorname{Int}(A))\right) \subseteq f^{-1}(A)$ for every closed set $A$ of $Y$.

(4) $w C l\left(f^{-1}(\operatorname{Int}(C l(B)))\right) \subseteq f^{-1}(C l(B))$ for every set $B$ of $Y$.

(5) $f^{-1}(\operatorname{Int}(B)) \subseteq w \operatorname{Int}\left(f^{-1}(C l(\operatorname{Int}(B)))\right.$ for every set $B$ of $Y$.

(6) $w C l\left(f^{-1}(V)\right) \subseteq f^{-1}(C l(V))$ for every open subset $V$ of $Y$.

Proof. (1) $\Rightarrow(2)$ Let $V$ be an open subset in $Y$ and $x \in f^{-1}(V)$. There exists a $w$-open subset $U$ of $X$ containing $x$ such that $f(U) \subseteq C l(V)$. Since $x \in U \subseteq f^{-1}(C l(V)), x \in w \operatorname{Int}\left(f^{-1}(C l(V))\right)$. Hence

$$
x \in f^{-1}(V) \subseteq w \operatorname{Int}\left(f^{-1}(w C l(V))\right) .
$$


(2) $\Rightarrow(3)$ Let $A$ be a closed subset in $Y$. Then $Y-A$ in open in $Y$ and, by

$$
\begin{array}{r}
f^{-1}(Y-A) \subseteq w \operatorname{Int}\left(f^{-1}(C l(Y-A))\right)=w \operatorname{Int}\left(f^{-1}(Y-\operatorname{Int}(A))\right) \\
\subseteq X-w C l\left(f^{-1}(\operatorname{Int}(A))\right) .
\end{array}
$$

Thus $w C l\left(f^{-1}(\operatorname{Int}(A))\right) \subseteq f^{-1}(A)$.

$(3) \Rightarrow(4)$ Let $B$ be a subset of $Y$. Since $C l(B)$ is closed in $Y$, from (3), it follows $w C l\left(f^{-1}(\operatorname{Int}(C l(B)))\right) \subseteq f^{-1}(C l(B))$.

$(4) \Rightarrow(5)$ Let $B$ be a subset of $Y$. Then

$$
\begin{array}{r}
f^{-1}(\operatorname{Int}(B))=X-f^{-1}(C l(Y-B)) \subseteq X-w C l\left(f^{-1} \operatorname{Int}(C l(Y-B))\right) \\
=w \operatorname{Int}\left(f^{-1} C l(\operatorname{Int}(B))\right) .
\end{array}
$$

Thus we get the result.

$(5) \Rightarrow(6)$ Let $V$ be an open subset of $Y$. Suppose $x \notin f^{-1}(C l(V))$. Then $f(x) \notin C l(V)$ and so there exists an open set $U$ containing $f(x)$ such that $U \cap V=\emptyset$ and so $C l(U) \cap V=\emptyset$. By (5), $x \in f^{-1}(U) \subseteq w \operatorname{Int}\left(f^{-1}(C l(U))\right)$. Hence there exists an open set $G$ containing $x$ such that $x \in G \subseteq f^{-1}(C l(U))$. Since

$$
C l(U) \cap V=\emptyset
$$

and

$$
f(G) \subseteq C l(U), \quad G \cap f^{-1}(V)=\emptyset
$$

and so $x \notin w C l\left(f^{-1}(V)\right)$. Hence $w C l\left(f^{-1}(V)\right) \subseteq f^{-1}(C l(V))$.

$(6) \Rightarrow(1)$ Let $x \in X$ and $V$ an open set in $Y$ containing $f(x)$. Since $V=\operatorname{Int}(V) \subseteq \operatorname{Int}(C l(V))$, by $(6)$,

$$
\begin{aligned}
& x \in f^{-1}(V) \subseteq f^{-1}(\operatorname{Int}(C l(V)))=X-f^{-1}(C l(Y-C l(V))) \\
& \subseteq X-w C l\left(f^{-1}(Y-C l(V))\right)=m \operatorname{mnt}\left(f^{-1}(C l(V))\right) .
\end{aligned}
$$

Hence there exists a $w$-open subset $U$ in $X$ such that $x \in U \subseteq f^{-1}(C l(V))$.

We recall that a point $x$ of a topological space $X$ is said to be $\theta$-adherent of $A$ if $A \cap C l(V) \neq \emptyset$ for every open set $V$ containing $x$. The set of all $\theta$ adherent points of $A$ is called $\theta$-closure of $A$ [18] and is denoted by $C l_{\theta}(A)$. If $A=C l_{\theta}(A)$, then $A$ is called $\theta$-closed. The complement of a $\theta$-closed set is said to be $\theta$-open. It is shown in [18] that $C l(A)=C l_{\theta}(A)$ for every open set $A$ and $C l_{\theta}(B)$ is closed for every subset $B$ of $X$. 
Theorem 3.4. Let $f:\left(X, w_{\sigma}\right) \rightarrow(Y, \mu)$ be a function on an associated $w$-space $\left(X, w_{\sigma}\right)$ and a topological space $(Y, \mu)$. Then the following statements are equivalent:

(1) $f$ is weakly $W O$-continuous.

(2) $w C l\left(f^{-1}\left(\operatorname{Int}\left(C l_{\theta}(B)\right)\right)\right) \subseteq f^{-1}\left(C l_{\theta}(B)\right)$ for every set $B$ of $Y$.

(3) $w C l\left(f^{-1}(\operatorname{Int}(C l(B)))\right) \subseteq f^{-1}\left(C l_{\theta}(B)\right)$ for every set $B$ of $Y$.

(4) $w C l\left(f^{-1}(\operatorname{Int}(C l(G)))\right) \subseteq f^{-1}(C l(G))$ for every open subset $G$ of $Y$.

(5) $f(w C l(A)) \subseteq C l_{\theta}(f(A))$ for every set $A$ of $X$.

(6) $w C l\left(f^{-1}(B)\right) \subseteq f^{-1}\left(C l_{\theta}(B)\right)$ for every set $B$ of $Y$.

Proof. (1) $\Rightarrow(2)$ Let $B$ be any subset in $Y$; then $C l_{\theta}(B)$ is closed, by Theorem $3.3(3)$, we get the result.

$(2) \Rightarrow(3)$ It is obvious since $C l(B) \subseteq C l_{\theta}(B)$ for every subset $B$ of $Y$.

$(3) \Rightarrow(4)$ It is obvious since $C l(G)=C l_{\theta}(G)$ for every open subset $G$ of $Y$.

$(4) \Rightarrow(1)$ Since $G \subseteq \operatorname{Int}(C l(G))$ for every open set $G$ of $Y$, from Theorem $3.3(6)$, it follows $f$ is weakly $W O$-continuous.

$(1) \Rightarrow(5)$ Let $A$ be a subset of $X$. Let $x \in w C l(A)$ and $G$ be an open subset of $Y$ containing $f(x)$. Since $f$ is weakly $W O$-continuous, there exists a $w$-open set $U$ containing $x$ in $X$ such that $f(U) \subseteq C l(G)$. Since $x \in w C l(A)$, we have $U \cap A \neq \emptyset$ and so $\emptyset \neq f(U) \cap f(A) \subseteq C l(G) \cap f(A)$. Thus we have $f(x) \in C l_{\theta}(f(A))$.

$(5) \Rightarrow(6)$ Let $B$ be a subset of $Y$; then by (5), we have

$$
f\left(w C l\left(f^{-1}(B)\right)\right) \subseteq C l_{\theta}\left(f\left(f^{-1}(B)\right)\right) \subseteq C l_{\theta}(B)
$$

and so we get the result.

$(6) \Rightarrow(1)$ Let $B$ be a subset of $Y$; then by (6),

$$
\begin{aligned}
w C l\left(f^{-1}(\operatorname{Int}(C l(B)))\right) \subseteq f^{-1}\left(C l_{\theta}(\operatorname{Int}(C l(B)))\right) \\
\quad=f^{-1}(C l(\operatorname{Int}(C l(B)))) \subseteq f^{-1}(C l(B)) .
\end{aligned}
$$

Hence $f$ is weakly $W O$-continuous by Theorem 3.3 (4). 
Let $X$ be a $w$-space. Then $X$ is said to be $w-T_{2}[15]$ if for every two distinct points $x$ and $y$ in $X$, there exist two disjoint weak open sets $U$ and $V$ such that $x \in U$ and $y \in V$.

Let $X$ be a topological space. Then $X$ is said to be Urysohn if for every two distinct points $x$ and $y$ in $X$, there exist two open sets $U$ and $V$ such that $C l(U) \cap C l(V)=\emptyset$.

Theorem 3.5. Let $f:\left(X, w_{\sigma}\right) \rightarrow(Y, \mu)$ be a function on an associated $w$-space $\left(X, w_{\sigma}\right)$ and a topological space $(Y, \mu)$. If $f$ is a weakly $W O$-continuous injection and $Y$ is Urysohn, then $X$ is $w-T_{2}$.

Proof. Let $x_{1}$ and $x_{2}$ be two distinct elements in $X$, then $f\left(x_{1}\right) \neq f\left(x_{2}\right)$. There exist two open sets $U$ and $V$ in $Y$ containing $f\left(x_{1}\right), f\left(x_{2}\right)$, respectively, such that $C l(U) \cap C l(V)=\emptyset$. Since $f$ is weakly $W O$-continuous, there exist $w$-open sets $U_{1}, V_{2}$ containing $x_{1}, x_{2}$, respectively, such that $f\left(U_{1}\right) \subseteq C l(U)$, $f\left(V_{2}\right) \subseteq C l(V)$. It follows $U_{1} \cap V_{2}=\emptyset$. Hence $X$ is $w-T_{2}$.

Definition 3.6. Let $f:\left(X, w_{\sigma}\right) \rightarrow(Y, \mu)$ be a function on an associated $w$-space $\left(X, w_{\sigma}\right)$ and a topological space $(Y, \mu)$. We call $f$ has a strongly $W$ closed graph if for each $(x, y) \notin G(f)$, there exist a $w$-open set $U$ and an open set $V$ containing $x$ and $y$, respectively, such that $(U \times C l(V)) \cap G(f)=\emptyset$.

Lemma 3.7. Let $f:\left(X, w_{\sigma}\right) \rightarrow(Y, \mu)$ be a function on an associated $w$ space $\left(X, w_{\sigma}\right)$ and a topological space $(Y, \mu)$. Then $f$ has a strongly $W$-closed graph if for each $(x, y) \notin G(f)$, there exist a $w$-open set $U$ containing and an open set $V$ containing $x$ and $y$, respectively, such that $f(U) \cap C l(V)=\emptyset$.

Proof. Obvious.

Theorem 3.8. Let $f:\left(X, w_{\sigma}\right) \rightarrow(Y, \mu)$ be a function on an associated $w$-space $\left(X, w_{\sigma}\right)$ and a topological space $(Y, \mu)$. If $f$ is weakly $W O$-continuous and $Y$ is Urysohn, then $f$ has a strongly $W$-closed graph.

Proof. Let $(x, z) \notin G(f)$. Then $z \neq f(x)$ and since $Y$ is Urysohn, there exist two open sets $U$ and $V$ containing $z$ and $f(x)$, respectively, such that $C l(U) \cap C l(V)=\emptyset$. Since $f$ is weakly $W O$-continuous, there exists a $w$-open set $H$ containing $x$ such that $f(H) \subseteq C l(V)$. It implies $f(H) \cap C l(U)=\emptyset$. Hence $f$ has a strongly $W$-closed graph.

Theorem 3.9. Let $f:\left(X, w_{\sigma}\right) \rightarrow(Y, \mu)$ be a function on an associated $w$-space $\left(X, w_{\sigma}\right)$ and a topological space $(Y, \mu)$. If $f$ is a weakly $W O$-continuous injection with a strongly $W$-closed graph, then $X$ is $w$ - $T_{2}$. 
Proof. Let $x_{1}$ and $x_{2}$ be two distinct elements in $X$, then $f\left(x_{1}\right) \neq f\left(x_{2}\right)$. This implies that $\left(x_{1}, f\left(x_{2}\right)\right) \in(X \times Y)-G(f)$. Since $f$ has a strongly $W$-closed graph, there exist a $w$-open set $U$ and an open set $V$ containing $x_{1}$ and $f\left(x_{2}\right)$, respectively, such that $f(U) \cap C l(V)=\emptyset$. Since $f$ is weakly $W O$-continuous, there exist a $w$-open set $W$ containing $x_{2}$ such that $f(W) \subset C l(V)$. It implies $f(W) \cap f(U)=\emptyset$. Therefore $W \cap U=\emptyset$ and so $X$ is a $w-T_{2}$ space.

A subset $A$ of a $w$-space $\left(X, w_{X}\right)$ is called $W$-compact [15] relative to $A$ if every collection $\left\{U_{i}: i \in J\right\}$ of $w$-open subsets of $X$ such that $A \subset \cup\left\{U_{i}: i \in\right.$ $J\}$, there exists a finite subset $J_{0}$ of $J$ such that $A \subset \cup\left\{U_{i}: i \in J_{0}\right\}$. A subset $A$ of a $w$-space $\left(X, w_{X}\right)$ is said to be $W$-compact if $A$ is $W$-compact as a subspace of $X$.

A subset $A$ of a $w$-space $X$ is said to be quasi $H$-closed relative to $(Y, \mu)[18]$ if every collection $\left\{U_{i}: i \in J\right\}$ of open subsets of $X$ such that $A \subset \cup\left\{U_{i}: i \in J\right\}$, there exists a finite subset $J_{0}$ of $J$ such that $A \subset \cup\left\{C l\left(U_{i}\right): i \in J_{0}\right\}$.

Theorem 3.10. Let $f:\left(X, w_{\sigma}\right) \rightarrow(Y, \mu)$ be a function on an associated $w$-space $\left(X, w_{\sigma}\right)$ and a topological space $(Y, \mu)$. If $f$ is weakly $W O$-continuous and $A$ is a $W$-compact subset of $X$, then $f(A)$ is quasi $H$-closed relative to $(Y, \mu)$.

Proof. Let $\left\{V_{i}: i \in J\right\}$ be a cover of $f(A)$ by open subsets of $Y$. For each $x \in A$, there exists $i(x) \in J$ such that $f(x)=y \in V_{i(x)}$. Since $f$ is weakly $W O$ continuous, there exists a $w$-open set $U(x)$ containing $x$ such that $f(U(x)) \subseteq$ $C l\left(V_{i(x)}\right)$. The family $\{U(x): x \in A\}$ is a cover of $A$ by $w$-open sets in $X$. Since $A$ is $w$-compact, there is a finite subcover $\left\{U\left(x_{1}\right), U\left(x_{2}\right), \cdots, U\left(x_{n}\right)\right.$ : $\left.x_{j} \in A, j=1,2, \cdots, n\right\}$ such that $A \subseteq \cup U\left(x_{j}\right)$. Then

$$
f(A) \subseteq f\left(\cup U\left(x_{j}\right)\right) \subseteq \cup f\left(U\left(x_{j}\right)\right) \subseteq \cup C l\left(V_{i\left(x_{j}\right)}\right)
$$

$1 \leq j \leq n$

Thus $f(A)$ is quasi $\mathrm{H}$-closed relative to $(Y, \mu)$.

\section{References}

[1] P. Bhattacharyya, B.K. Lahiri, Semi-generalized closed sets in topology, Indian J. Math., 29, No. 3 (1987), 375-382.

[2] Á. Csázár, Generalized topology, generalized continuity, Acta Math. Hungar., 96 (2002), 351-357. 
[3] J. Dontchev, H. Maki, On sg-closed sets and semi- $\lambda$-closed sets, QEBA in General Topology, 15 (1997), 259-266.

[4] D.C. Kent, W.K. Min, Neighborhood spaces, International Journal of Mathematics and Mathematical Sciences, 32, No. 7 (2002), 387-399.

[5] N. Levine, Generalized closed sets in topology, Rend. Cir. Mat. Palermo, 19 (1970), 89-96.

[6] N. Levine, Semi-open sets and semi-continuity in topological spaces, Ams. Math. Monthly, 70 (1963), 36-41.

[7] H. Maki, J. Umehara, T. Noiri, Every topological space is pre- $T_{\frac{1}{2}}, M e m$. Fac. Sci. Kochi Univ. Ser. A, 17 (1996), 33-42.

[8] H. Maki, R. Devi, K. Balachandran, Associated topologies of generalized $\alpha$-closed sets and $\alpha$-generalized closed sets, Mem. Fac. Sci. Kochi Univ. Ser. A, 15 (1994), 51-63.

[9] Maki, R. Devi, K. Balachandran, Generalized $\alpha$-closed maps and $\alpha$ generalized closed maps, Indian J. Pure Appl. Math., 29, No. 1 (1998), $37-49$.

[10] A.S. Mashhour, M.E. Abd El-Monsef, S.N. El-Deeb, On precontinuous and weak precontinuous mappings, Proc. Math. and Phys. Soc. Egypt, 53 (1982), 47-53.

[11] W.K. Min, Some results on generalized topological spaces and generalized systems, Acta Math. Hungar., 108 (1-2) (2005), 171-181.

[12] W.K. Min, On weak neighborhood systems and weak neighborhood spaces, Acta Math. Hungar., (2008), doi: 10.1007/s10474-008-7205-8.

[13] W.K. Min, Y.K. Kim, On weak structures and w-spaces, Far East Journal of Mathematical Sciences, Accepted.

[14] W.K. Min, Y.K. Kim, $W O$-continuity and $W K$-continuity on associated $w$ spaces, International Journal of Pure and Applied Mathematics, Accepted.

[15] W.K. Min, Y.K. Kim, On product weak structures and product $w$-spaces, International Journal of Mathematical Analysis, Accepted.

[16] O. Njastad, On some classes of nearly open sets, Pacific Journal of Mathematics, 15, No. 3 (1964), 961-970. 
[17] F. Siwiec, On defining a space by a weak base, Pacific Journal of Mathematics, 52, No. 1 (1974), 351-357.

[18] N.V. Velicko, H-closed topological spaces, Amer. Math. Soc. Transl., 78 (1968), 103-118. 